\title{
Day-to-Day Scheduling Travel Time Adjustment Behavior and Simulation
}

\author{
Hua-Min Li, ${ }^{1,2}$ David Z. W. Wang, ${ }^{2}$ Hai-Jun Huang, ${ }^{3}$ and Xiao-Jun Yu ${ }^{4}$ \\ ${ }^{1}$ College of Automation, Chongqing University, Chongqing 400044, China \\ ${ }^{2}$ School of Civil and Environmental Engineering, Nanyang Technological University, Singapore 639798 \\ ${ }^{3}$ School of Economics and Management, Beihang University, Beijing 100191, China \\ ${ }^{4}$ School of Mathematics \& Statistics, Guizhou University of Finance \& Economics, Guiyang 550004, China \\ Correspondence should be addressed to Hua-Min Li; huaminli@cqu.edu.cn
}

Received 19 February 2014; Revised 6 April 2014; Accepted 13 April 2014; Published 30 April 2014

Academic Editor: X. Zhang

Copyright ( 2014 Hua-Min Li et al. This is an open access article distributed under the Creative Commons Attribution License, which permits unrestricted use, distribution, and reproduction in any medium, provided the original work is properly cited.

\begin{abstract}
This paper proposes a modeling framework to study the day-to-day scheduling travel time adjustment behavior on the base of past experiences. Scheduling travel time is defined as the difference between the requested arrival time and the departure time. Mathematical equations are established to formulate every traveler's dynamic adjustment on his/her departure time. In the adjustment process, the last day's scheduling time is an essential component, while the numbers of late arrivals, punctual arrivals, and early arrivals in a previous day are used to reflect the past experiences. Simulation results are presented to illustrate the effectiveness of the proposed modeling framework.
\end{abstract}

\section{Introduction}

Travel time has always been a fundamental component of the transportation investigation and has been applied in various forms. A large number of researches and literature reviews are concerned with the formula and estimation of travel time, which constitutes the essential work of studying various traffic assignments based on the principles of user equilibrium, system optimization, and their stochastic and/or dynamic counterparts. Meanwhile, travel time is an important index to evaluate the performance of a transportation system and hence is regularly surveyed in almost all cities. Travel time is also information widely used in the advanced traffic management systems and in-vehicle route guidance systems.

Travel time can be estimated by using different approaches, depending upon the applications with different purposes. The point delay models are commonly used to estimate the travel times of signalized links (Webster [1]; Allsop [2]). In the Highway Capacity Manual [3], the average travel time of a link is calculated as the sum of the link running time and the intersection delay which is given by a deterministic point delay model. Skabardonis and Geroliminis [4] proposed an analytical model to estimate the travel times of arterial streets, using the 15-30-second flow and the occupancy data provided by loop detectors and traffic signal settings. These studies mainly focused on the estimation of average travel time.

Travel time is affected by some unknown factors and is thus stochastic, especially in the complex urban environment. This makes the investigation of travel time uncertainty become a hot topic in recent years. Guo et al. [5] proposed a multistate model which is employed to fit a mixture of Gaussian distributions into travel time observations of an expressway corridor. Each normal distribution is associated with an underlying traffic state providing quantitative uncertainty evaluation. The multistate mixture model leads to a better fitting, revealing that travel time distribution usually has more than one mode which is entirely dependent on the time horizon of the study, the demand, the topology, and others. Using multistate models, a recent work by Park et al. [6] tried to quantify the impact of traffic incidents. Feng et al. [7] 


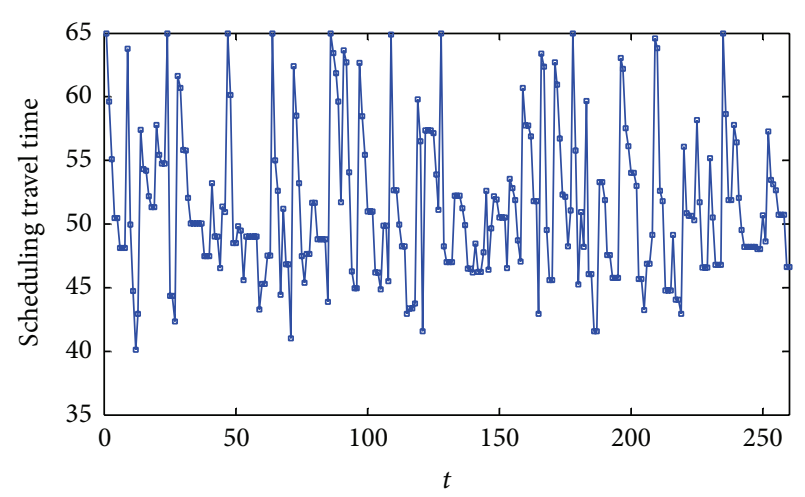

FIgURE 1: Day-to-day scheduling travel time when Toltime = 5 and $R v=(b-a) / 6$.

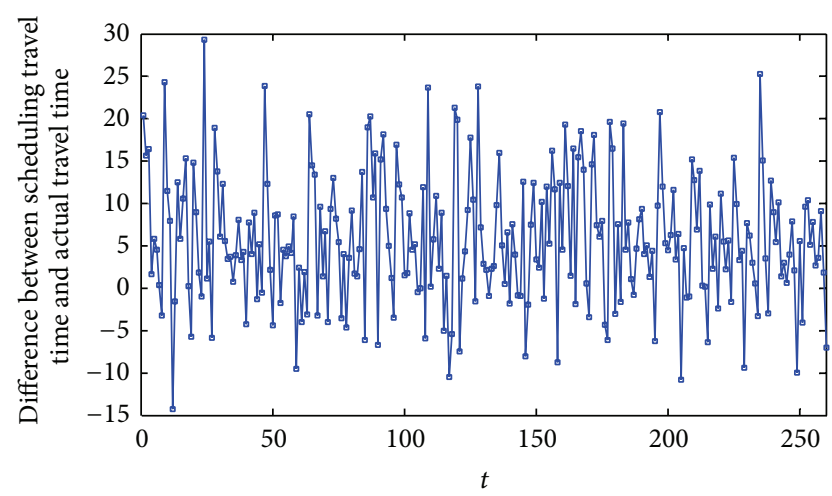

FIGURE 2: Day-to-day difference between scheduling travel time and actual travel time when Toltime $=5$ and $R v=(b-a) / 6$.

conducted a similar investigation, utilizing mixtures of normal distributions to estimate mean travel times for arterial routes.

As mentioned above, the actual travel time in an urban traffic environment is highly stochastic and time-dependent due to random fluctuations in interruptions caused by traffic control devices, weather conditions, and day-to-day events, such as vehicle stall, minor accident, traffic signal, bus stop, merge and diverge bottleneck, truck and bus platoon, and train-crossing. Considering these factors is essential for improving the estimation accuracy and expanding the relevant studies. It has been increasingly recognized that the actual travel time should be assumed to be stochastic.

On the other hand, travelers, especially commuters, learn the travel times based on the past experiences and then determine their departure times. They realize that traffic participants, vehicles, and such facilities as roads and signals are the main components of traffic system. Various factors which affect the operation of the components would influence the final travel time. For instance, different drivers and road conditions could result in large variation of travel times. Even in the same time interval and on the same link, different vehicles can have quite different travel times (Li and McDonald [8]). Travelers recognize that the actual travel time is stochastic and should adjust their departure time according

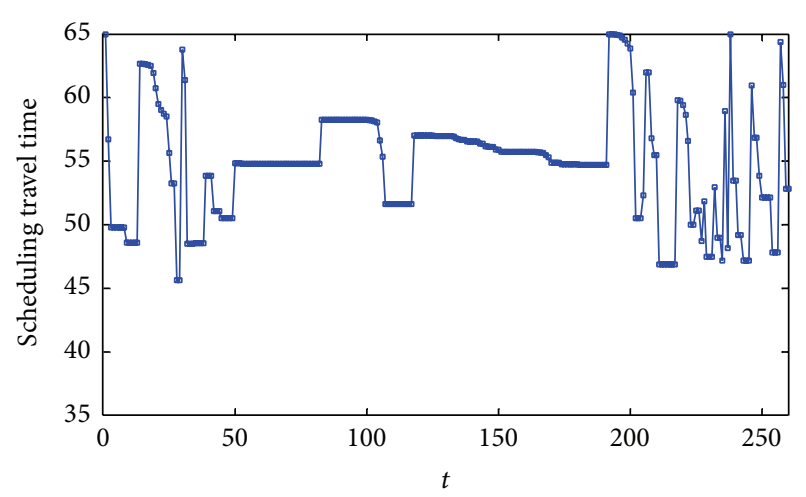

Figure 3: Day-to-day scheduling travel time when Toltime = 10 and $R v=(b-a) / 6$ (scenario 1$)$.

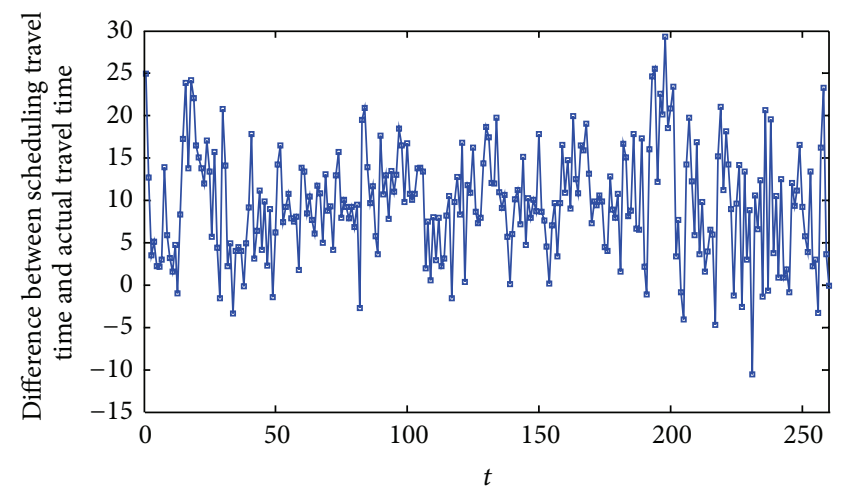

FIGURE 4: Day-to-day difference between scheduling travel time and actual travel time when Toltime $=10$ and $R v=(b-a) / 6$ (scenario $1)$.

to their past experiences on late arrival, punctual arrival, and early arrival. More importantly, due to the randomness of human choices, these adjustments should not be in general deterministic but random.

In Section 2 of this paper, we make a synthetic consideration about the scheduling travel time and the actual travel time. In Section 3, a model for formulating the day-to-day scheduling travel time adjustment behavior is proposed. In Section 4, simulation results are presented to illustrate the effectiveness of the proposed model. Section 5 concludes the paper.

\section{Specifications of Scheduling Travel Time and Actual Travel Time}

We define the scheduling travel time, $T P_{t}$, as the time consumption on day $t$ required for traveling from an origin to a destination (e.g., from a commuter's residence to his/her working place) and exactly arriving at the requested time. Thus, the scheduling travel time $T P_{t}$ is equal to the requested arrival time minus the departure time. Obviously the scheduling travel time should vary within a certain range of time defined by an upper bound $b$ and a lower bound $a$ that is corresponding to the free-flow travel time. 


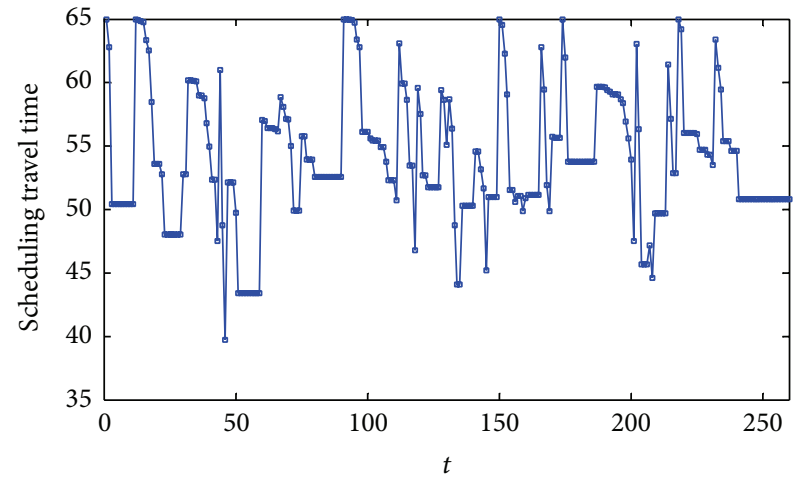

FIGURE 5: Day-to-day scheduling travel time when Toltime = 10 and $R v=(b-a) / 6$ (scenario 2$)$.

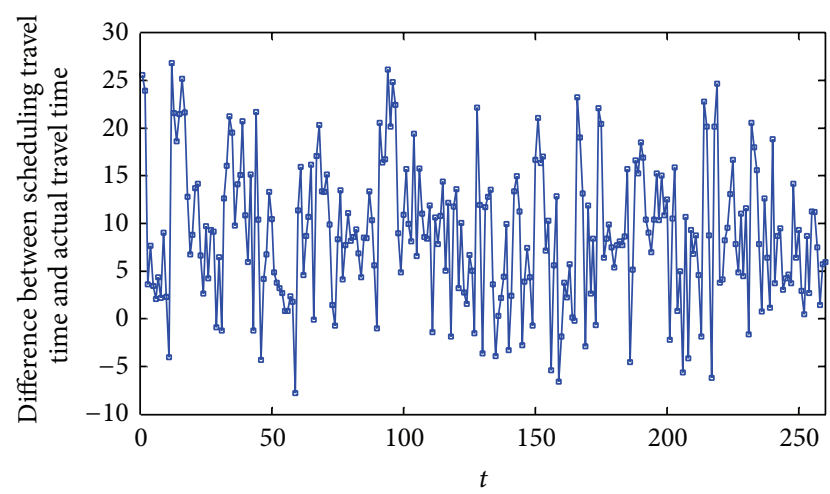

FIGURE 6: Day-to-day difference between scheduling and actual travel times when Toltime $=10$ and $R v=(b-a) / 6$ (scenario 2).

In reality, it is easily observed that a commuter will modify his departure time on day $t$ if he arrives lately or early on day $t-1$. This modification should simultaneously take into account his past experiences, such as the numbers of late arrivals, punctual arrivals, and early arrivals in previous days. A commuter is willing to retain his departure time on day $t$ if he arrives on time on day $t-1$. In this regard, a commuter will adjust his departure time whenever he arrived late or early. The adjustment process and its magnitude should depend on each commuter's preferences and recallable experience. However, the mechanism by which each individual handles his experiences and carries out the adjustment is not known (Chang and Mahmassani [9]). Therefore, it is reasonable to assume this adjustment to be a random variant related to the past experiences. Consequently, according to the preceding analysis, the scheduling travel time on day $t$ can be defined as the scheduling travel time on day $t-1$ plus a corresponding random variable that grounds on the last situation.

The actual travel time, denoted as $T R_{t}$, is the time a commuter actually travels from his origin to the destination on day $t$. As mentioned at the foregoing paragraph, numerous factors affect the actual travel time. The actual travel time is highly stochastic. Thus, we can presume that the actual travel time is a random variable which has a lower bound $a$ given by the free-flow travel time but has no upper bound.

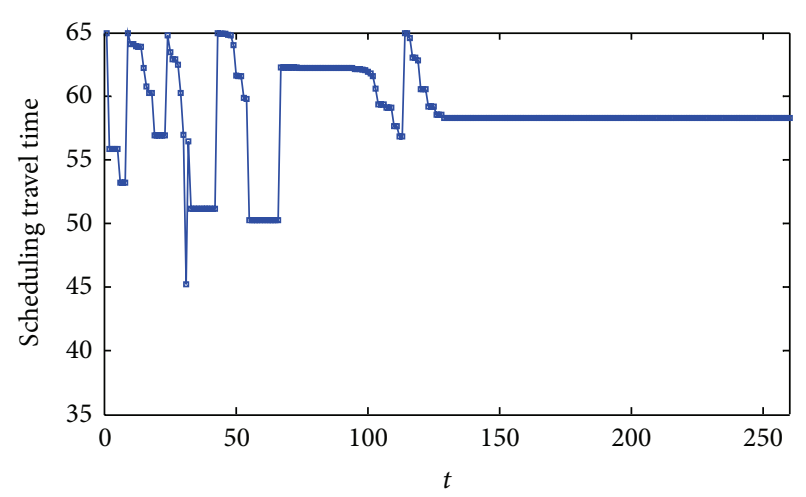

FIgURE 7: Day-to-day scheduling travel time when Toltime = 15 and $R v=(b-a) / 6$.

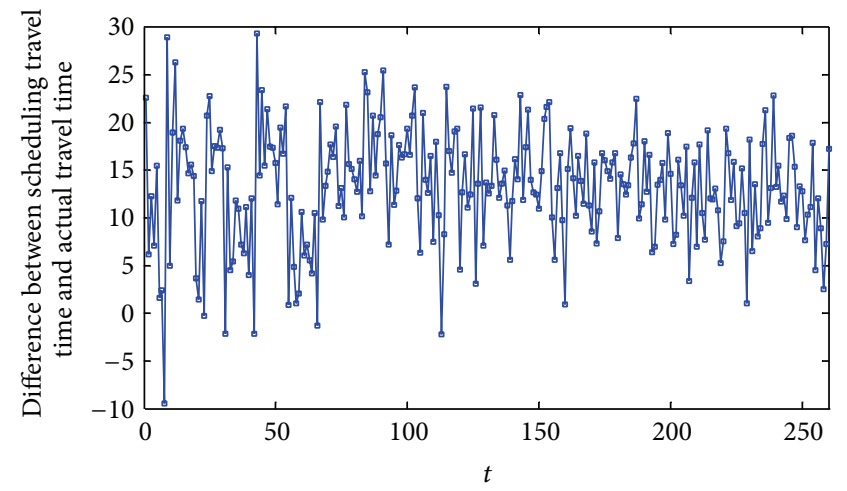

FIGURE 8: Day-to-day difference between scheduling travel time and actual travel time when Toltime $=15$ and $R v=(b-a) / 6$.

\section{Day-to-Day Scheduling Travel Time Adjustment Behavior}

In this study, we propose a model that incorporates dynamic and randomness into consumer choice of departure time. In our day-to-day decision-making framework for scheduling travel time (corresponding to departure time), a commuter will adjust his scheduling travel time whenever he experienced the difference between last scheduling travel time and last actual travel time. The model allows for an increase and a decrease of the scheduling travel time in a particular fashion that is interrelated with the numbers of previous late arrivals, punctual arrivals, and early arrivals. If the commuter arrives late, he will be induced to increase the scheduling travel time on the subsequent day. If the commuter arrives early, he will be more likely to decrease the scheduling travel time. Otherwise, the commuter will retain the scheduling travel time on the subsequent day if he arrives on time at present day.

An assumption is made that not only does the researcher not observe all factors in the consumer's adjustment, but also, even in the same situation, the same commuter can make quite different adjustment. Therefore, a random term is supposed to incorporate into the adjustment to represent this unobserved component. On the other hand, to reflect 


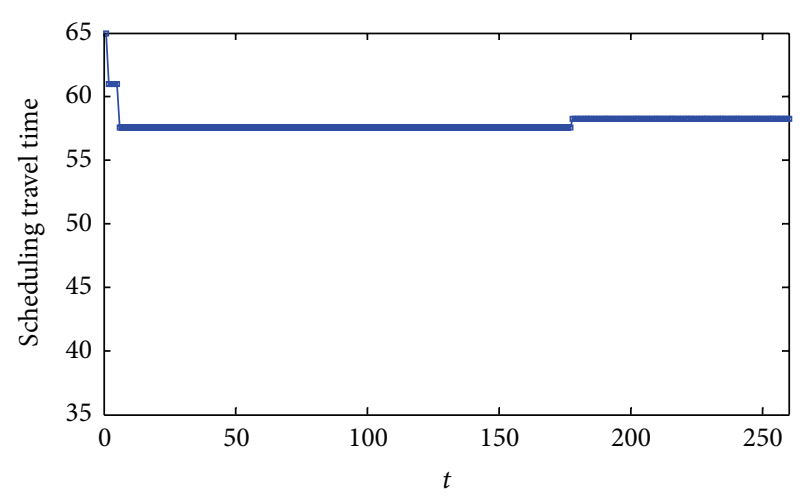

Figure 9: Day-to-day scheduling travel time when Toltime $=20$ and $R v=(b-a) / 6$.

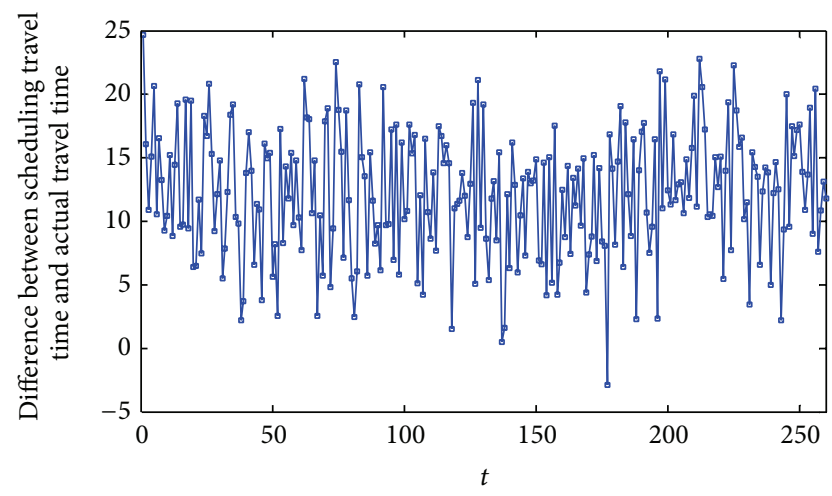

Figure 10: Day-to-day difference between scheduling travel time and actual travel time when Toltime $=20$ and $R v=(b-a) / 6$.

the commuter's past experiences more appropriately, the approach considers the numbers of late arrivals, punctual arrivals, and early arrivals on a previous day.

Summing up the above description and analysis to mathematical formulation, the scheduling travel time of day $t$ is given by

$$
\begin{aligned}
T P_{t}= & I_{L, t-1} \\
& \cdot \min \left(T P_{t-1}+\left(1+\frac{e^{N_{L t}}}{e^{N_{L t}}+e^{N_{E t}}+e^{N_{P t}}}\right)\right. \\
& \left.\cdot\left[(b-a) \cdot\left(1-\sqrt{2\left(1-\mu_{1}\right)}\right)\right], b\right) \\
+ & I_{E, t-1} \cdot \max \left(T P_{t-1}+\frac{e^{N_{E t}}}{e^{N_{L t}+e^{N_{E t}}+e^{N_{P t}}}}\right. \\
& \left.\cdot\left[\left(T P_{t-1}-\mathrm{TR}_{t-1}\right) \cdot\left(\sqrt{2 \mu_{2}}-1\right)\right], a\right) \\
+ & I_{P, t-1} \cdot T P_{t-1},
\end{aligned}
$$

where $T P_{t}$ is the scheduling travel time selected by the commuter on day $t, T R_{t}$ is the actual travel time experienced

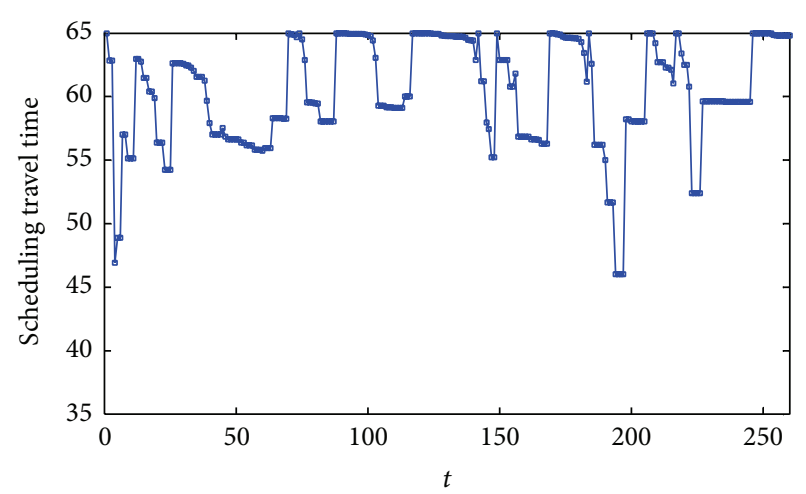

FIGURE 11: Day-to-day scheduling travel time, when Toltime = 15 and $R v=(b-a) / 3$.

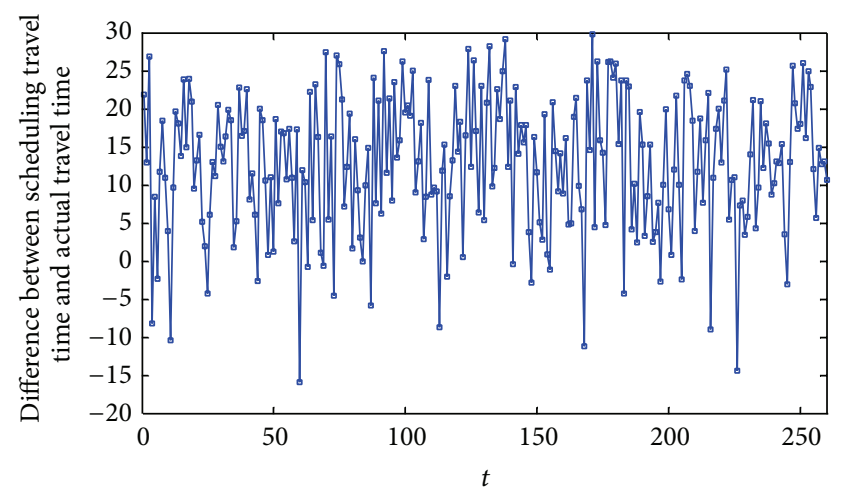

FIGURE 12: Day-to-day difference between scheduling travel time and actual travel time when Toltime $=15$ and $R v=(b-a) / 3$.

by the commuter on day $t, I_{L, t}$ is an indicator parameter whose value is one if the commuter is late for his commute on day $t$ (i.e., $T P_{t}<T R_{t}$ ), otherwise zero, and $I_{P, t}$ is another indicator parameter whose value is one if the commuter arrives at destination punctually on day $t$, otherwise zero. Note that the punctuality here means that the commuter arrives before the requested arrival time within a tolerable time range. This says that $0 \leq T P_{t}-T R_{t} \leq$ Toltime, where Toltime is the longest time that is acceptable for the commuter to arrive early, that is, the maximum time the commuter can tolerate if he arrives earlier than the requested time. $I_{E, t}$ is an indicator parameter whose value is one if the commuter arrives early on day $t$; that is, $T P_{t}-$ $T R_{t} \geq$ Toltime. $N_{L t}, N_{P t}$, and $N_{E t}$ are the numbers of late arrivals, punctual arrivals, and early arrivals before the day $t$, respectively. $\mu_{1}$ is a random parameter following a uniform distribution between 0.5 and $1 ; \mu_{2}$ is also a random parameter distributed uniformly between 0 and 0.5. $a$ and $b$ are, respectively, the lower and upper bounds of the scheduling travel time. Note again that the actual travel time $T R_{t}$ is assumed to be a random variable that ranges from $a$ to infinity governed by a normal density distribution with mean $R m$ and standard deviation $R v$.

Model (1) is explained as follows. If the commuter arrives punctually on day $t-1$, he spontaneously remains with 


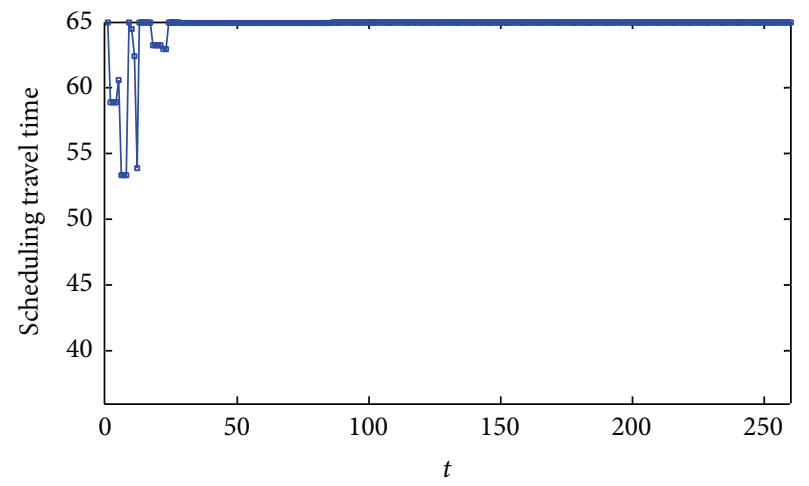

Figure 13: Day-to-day scheduling travel time when Toltime $=20$ and $R v=(b-a) / 3$.

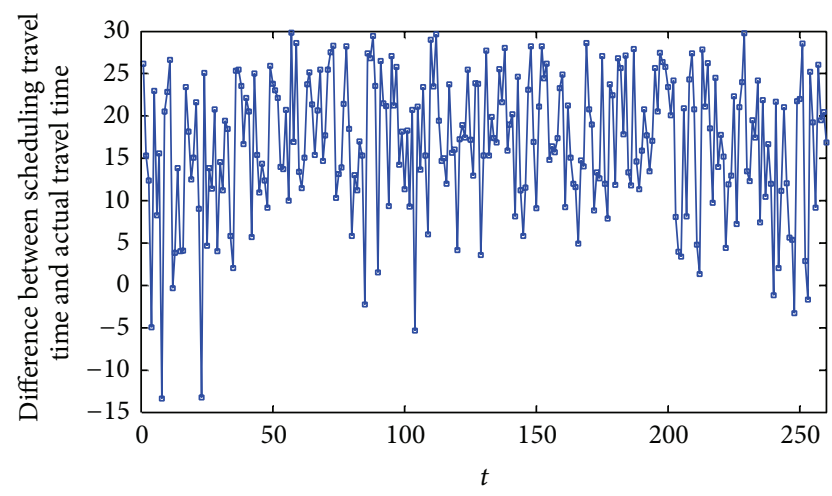

Figure 14: Day-to-day difference between scheduling travel time and actual travel time when Toltime $=20$ and $R v=(b-a) / 3$.

the same scheduling travel time on day $t$. If the commuter arrives late on day $t-1$, he naturally intends to depart earlier on day $t$ than on day $t-1$. So, the scheduling travel time should increase on day $t$, which is reflected by

$$
\begin{aligned}
T P_{t-1}+ & \left(1+\frac{e^{N_{L t}}}{e^{N_{L t}}+e^{N_{E t}}+e^{N_{P t}}}\right) \\
\cdot & {\left[(b-a) \cdot\left(1-\sqrt{2\left(1-\mu_{1}\right)}\right)\right] . }
\end{aligned}
$$

In the above, the second item represents the extension of the scheduling travel time for considering the commuter's past experiences and randomness. $\left(1+\left(e^{N_{L t}} /\left(e^{N_{L t}}+e^{N_{E t}}+e^{N_{P t}}\right)\right)\right)$ states that the numbers of late arrivals, punctual arrivals, and early arrivals are involved into the adjustment. $(b-a)$ denotes the potential interval of the adjustment and $\left(1-\sqrt{2\left(1-\mu_{1}\right)}\right)$ displays the randomness characteristics of human choices. On the other hand, it is impossible for a commuter to leave home too early. For instance, if a commuter is required to arrive at a destination at 8:00 am, he or she certainly will not leave home before 1:00 am. Then, $T P_{t}$ should logically have an upper bound. Therefore, when the commuter arrives late on day $t-1, \min \left(T P_{t-1}+\left(1+\left(e^{N_{L t}} /\left(e^{N_{L t}}+e^{N_{E t}}+\right.\right.\right.\right.$ $\left.\left.\left.\left.e^{N_{P t}}\right)\right)\right) \cdot\left[(b-a) \cdot\left(1-\sqrt{2\left(1-\mu_{1}\right)}\right)\right], b\right)$ can correctly depict the adjustment of scheduling travel time on day $t$. If the

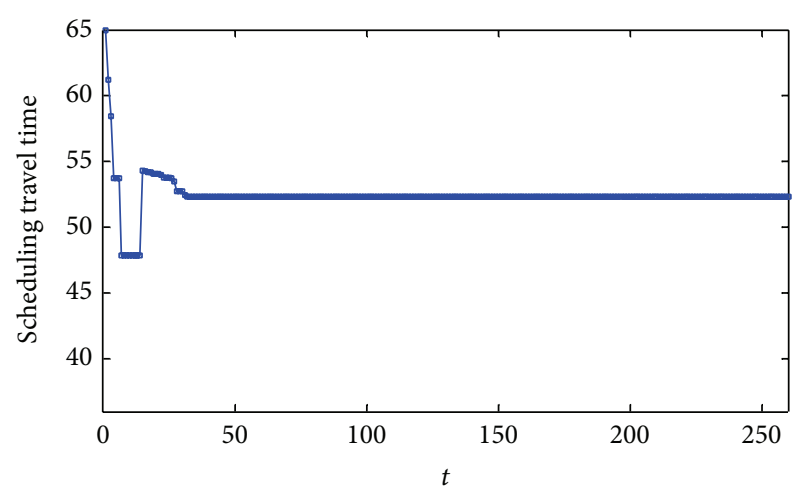

FIGURE 15: Day-to-day scheduling travel time when Toltime $=10$ and $R v=(b-a) / 12$.

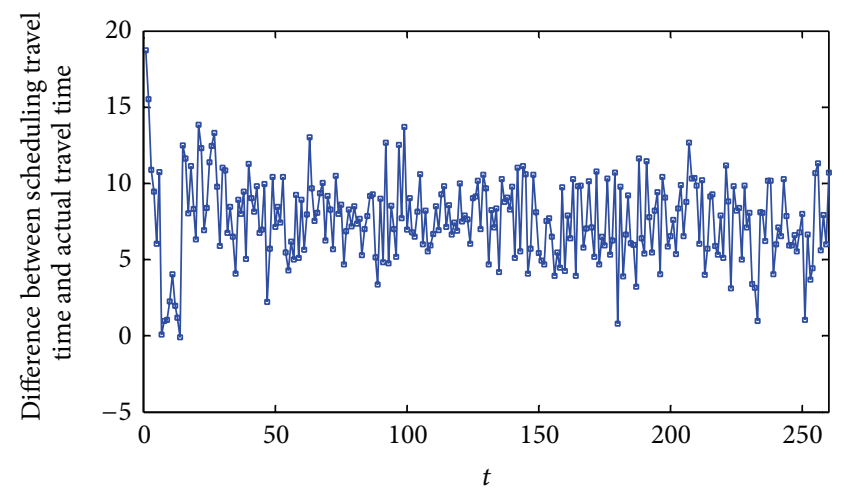

FIGURE 16: Day-to-day difference between scheduling travel time and actual travel time when Toltime $=10$ and $R v=(b-a) / 12$.

commuter arrives early, the second item in (1) gives the adjustment on scheduling travel time. The major difference between the first and the second items of the equation is that the adjustment of scheduling travel time in the case of arriving late mainly considers $(b-a)$ for avoiding penalty but $T P_{t-1}-T R_{t-1}$ in the case of arriving early.

Equation (1) can be rewritten as

$$
\begin{aligned}
& T P_{t}= I\left(T P_{t-1}<T R_{t-1}\right) \\
& \cdot \min \left(T P_{t-1}+\left(1+\frac{e^{N_{L t}}}{e^{N_{L t}}+e^{N_{E t}}+e^{N_{P t}}}\right)\right. \\
&\left.\left.\cdot\left[(b-a) \cdot\left(1-\sqrt{2\left(1-\mu_{1}\right.}\right)\right)\right], b\right) \\
&+I\left(T P_{t-1}-T R_{t-1} \geq \text { Toltime }\right) \\
& \cdot \max \left(T P_{t-1}+\frac{e^{N_{E t}}}{e^{N_{L t}}+e^{N_{E t}}+e^{N_{P t}}}\right. \\
&\left.\quad \cdot\left[\left(T P_{t-1}-T R_{t-1}\right) \cdot\left(\sqrt{2 \mu_{2}}-1\right)\right], a\right) \\
&+I\left(0 \leq T P_{t-1}-T R_{t-1} \leq \text { Toltime }\right) \cdot T P_{t-1},
\end{aligned}
$$


where $I(\cdot)$ is a step function whose value is one if the event in the parenthesis occurs, zero otherwise.

\section{Simulation}

This section aims at illustrating the model's capability of depicting the commuters' scheduling travel time adjustment behavior and revealing some properties of the adjustment behavior by changing the parameter values. The evolution of the day-to-day scheduling travel times and the trend of the day-to-day departure times will be presented. The maximum value of the time index $t$ is set to be 260 (basically the number of working days in one year).

Suppose the lower bound of the scheduling travel time $a$ is 35 minutes and the upper bound $b$ is 65 minutes. The actual travel time is assumed to be a random variable that ranges from 35 to infinity with density the same as the normal density, with mean $R m$ and standard deviation $R v$ within this range. Because there exist differences between actual travel time distribution and perceived travel time distribution (Chen et al. [10]), the mean of the actual travel time distribution is generally less than the mean of the perceived travel time distribution. Consequently, if the scheduling travel time and the actual travel time are both considered to be random variables, the mean of the actual travel time should be less than the mean of the scheduling travel time distribution. For simplicity, $R m$ is assumed to be less than $0.5(a+b)-5$ in this study. Furthermore, some events (e.g., wide geographical bumper-to-bumper traffic jams) are with small probability and can be handled by the three-sigma rule or an empirical rule which states that nearly all values of a random variable are in a range covering 3 standard deviations from the mean. So, $R v$ can be logically presumed to take $(b-a) / 6$ (this is from $0.5(a+b)-a=3 R v)$.

First, let Toltime be equal to 5 . Figure 1 displays the scheduling travel time and Figure 2 the difference between scheduling travel time and actual travel times. It can be seen that there are wide variations concerning the changes of scheduling travel time against day index. This implies that the departure time is selected nearly at random. Simulation results show that the numbers of late arrivals, punctual arrivals, and early arrivals in these 260 days are 56, 73, and 131, respectively. So, wide variations indeed occur. The reason is that the value of Toltime is too small, which makes commuters obligated to arrive punctually. Therefore, small tolerance leads to big variations in departure time.

Second, let Toltime be 10. Figures 3 to 6, respectively, exhibit two scenarios that are mostly likely to occur under the assumptions; Toltime $=10$ and $R v=(b-a) / 6$. Because the tolerable time, Toltime, is increased, there are relatively small variations in the scheduling travel time and a relatively narrow range of the difference between scheduling travel time and actual travel time, compared to the results shown in Figures 1 and 2. As shown in Figures 3 and 4, the scheduling times are established in a relatively consistent daily schedule but still disrupted by small events due to the randomness of actual travel time. Accordingly, the numbers of late arrivals, punctual arrivals, and early arrivals are around 19, 121, and
120, respectively. Nevertheless, the similar result shown in Figures 1 and 2 likely occurs in Figures 5 and 6, where the numbers of late arrivals, punctual arrivals, and early arrivals are around 30,122, and 108, respectively. Both scenarios may occur, which depends on the actual travel time sourced from reality.

Third, further enlarge Toltime to 15. As shown in Figures 7 and 8, the scheduling travel time is likely to be fixed and be disrupted occasionally, and the difference between scheduling travel time and actual travel time is not as large as that when Toltime is small. In addition, the number of late arrivals is only around 6; the number of punctual arrivals stands at more than 150 .

Fourth, let Toltime be 20. Figures 9 and 10 show the simulation results. It can be seen that the scheduling travel time is almost fixed and hardly disrupted. Due to the use of a large Toltime, the number of late arrivals is very small, but the number of punctual arrivals is almost up to 244 .

In the previous analyses, we set $R v$ as $(b-a) / 6$. When $R v$ is changed to $(b-a) / 3$, there are two representative scenarios. Let Toltime be equal to 15 and 20, respectively. As the standard deviation of actual travel time distribution increases, the results shown in Figures 11 and 12 are analogues to those in Figures 5 and 6 (where Toltime $=10$ and $R v=$ $(b-a) / 6)$, respectively. The numbers of late arrivals, punctual arrivals, and early arrivals in these two cases are likely to be equal, respectively. The appearance exhibited in Figure 13 is like that in Figure 9, but the scheduling travel time in the former approaches 65 while 58 in the later, and there exist significant differences between the numbers of late arrivals, punctual arrivals, and early arrivals in these two cases (in the case of Figures 13 and 14, the number of punctual arrivals is about 144).

Finally, let $R v$ be $(b-a) / 12$, which means that the actual travel time varies a little. The scheduling travel time approaches 53 even if Toltime is 10, as shown in Figure 15. The difference between scheduling travel time and actual travel time varies within a narrow range, as exhibited in Figure 16. The reason is that the actual travel time remains at a stable state, so that commuters easily adjust their scheduling travel times to match the actual travel time. The number of punctual arrivals is about 209 .

\section{Conclusions}

In this paper, the day-to-day scheduling travel time adjustment behavior (also, the day-to-day departure time adjustment behavior) is studied. A modeling framework is constructed to formulate the adjustment. The experiences of the last day's arrival (late arrival, punctual arrival, or early arrival) are used to influence today's behavior. The randomness of the commuters' behavior is also considered. Simulation results support the effectiveness of the modeling framework. Two parameters, that is, the maximum time that a commuter can tolerate if he or she arrives earlier than the requested time and the standard deviation of the actual travel time distribution, are numerically investigated. Results show that they have big impacts on scheduling travel time adjustment behavior. 


\section{Conflict of Interests}

The authors declare that there is no conflict of interests regarding the publication of this paper.

\section{Acknowledgments}

This study has been supported by the National Natural Science Foundation of China (Grant no. 71201178) and the Fundamental Research Funds for the Central Universities (Grant no. CDJZR12170009). And it was partly funded by the National Natural Science Foundation of China (Grant no. 71161005) and the Nomarch Foundation of Guizhou Province (Grant no. 2011067).

\section{References}

[1] F. Webster, "Traffic signal settings," Road Research Technical Paper no. 39, Road Research Laboratory, Berkshire, UK, 1958.

[2] R. E. Allsop, "Delay at fixed time traffic signals-I: theoretical analysis," Transportation Science, vol. 6, no. 3, pp. 260-285, 1972.

[3] National Research Council, Highway Capacity Manual, Transportation Research Board, Washington, DC, USA, 2000.

[4] A. Skabardonis and N. Geroliminis, "Real-time estimation of travel times on signalized arterials," in Proceedings of the 16th International Symposium on Transportation and Traffic Theory, pp. 387-406, University of Maryland, College Park, Md, USA, 2005.

[5] F. Guo, H. Rakha, and S. Park, "Multistate model for travel time reliability," Transportation Research Record, vol. 2188, pp. 46-54, 2010.

[6] S. Park, H. Rakha, and F. Guo, "Multi-state travel time reliability model: impact of incidents on travel time reliability," in Proceedings of the 14th IEEE International Intelligent Transportation Systems Conference (ITSC '11), pp. 2106-2111, Washington, DC, USA, October 2011.

[7] Y. Feng, G. Davis, and J. Hourdos, "Arterial travel time characterization and real-time traffic condition identification using GPS-equipped probe vehicles," in Proceedings of the 90th Transportation Research Board Annual Meeting, Washington, DC, USA, 2011.

[8] Y. Li and M. McDonald, "Link travel time estimation using single GPS equipped probe vehicle," in Proceedings of the 5th IEEE International Conference on Intelligent Transportation Systems, pp. 932-937, Singapore, 2002.

[9] G.-L. Chang and H. S. Mahmassani, "Travel time prediction and departure time adjustment behavior dynamics in a congested traffic system," Transportation Research B: Methodological, vol. 22, no. 3, pp. 217-232, 1988.

[10] A. Chen, Z. Zhou, and W. H. K. Lam, "Modeling stochastic perception error in the mean-excess traffic equilibrium model," Transportation Research B: Methodological, vol. 45, no. 10, pp. 1619-1640, 2011. 


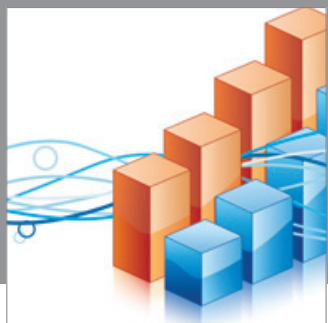

Advances in

Operations Research

mansans

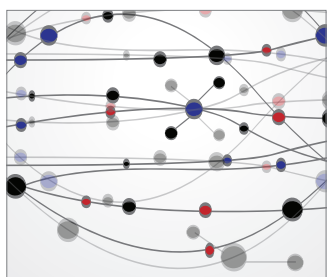

The Scientific World Journal
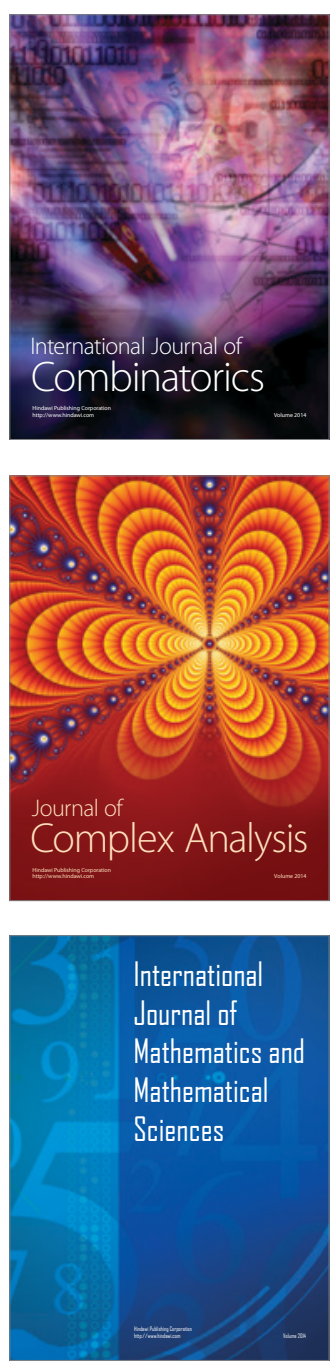
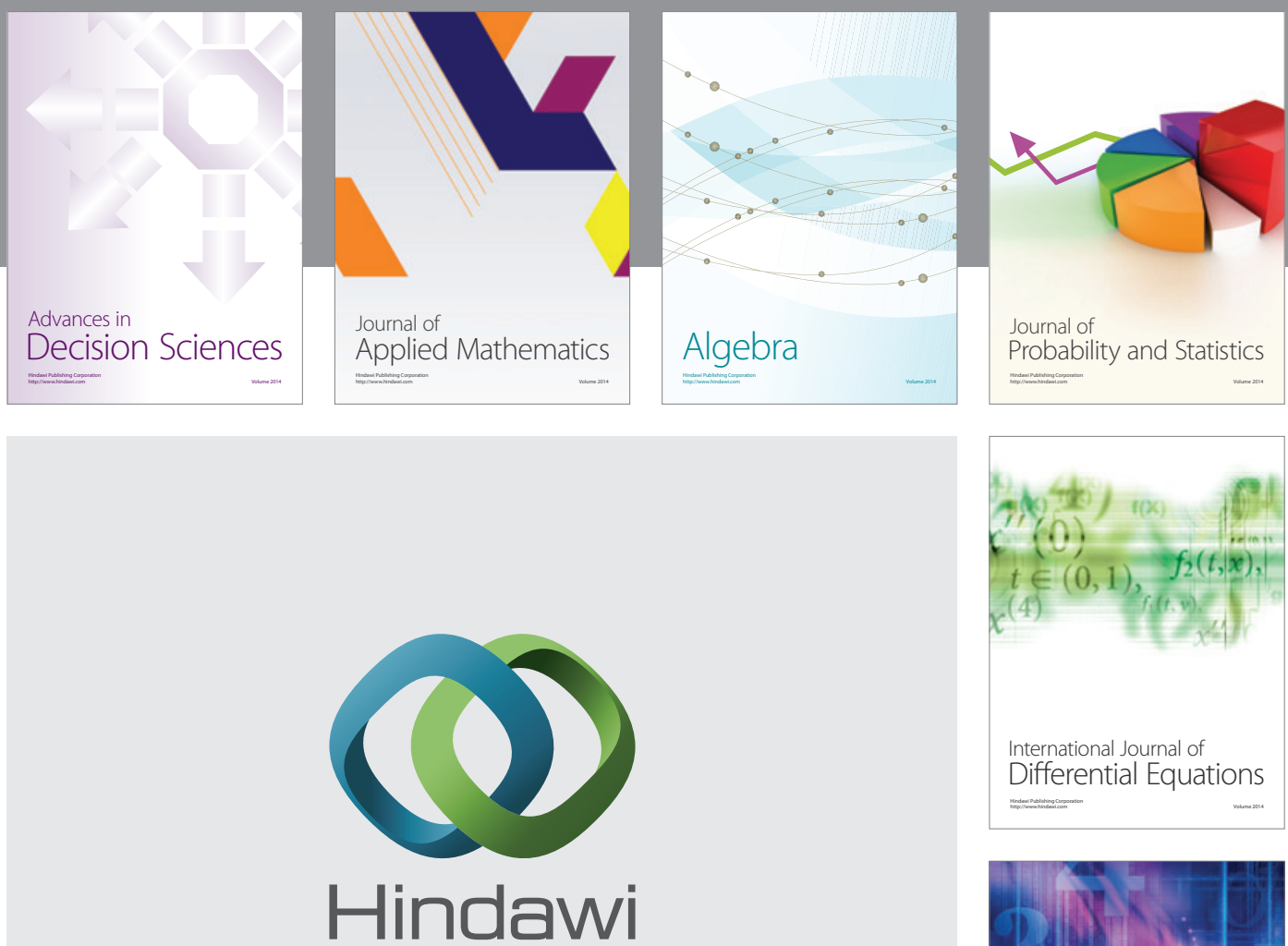

Submit your manuscripts at http://www.hindawi.com
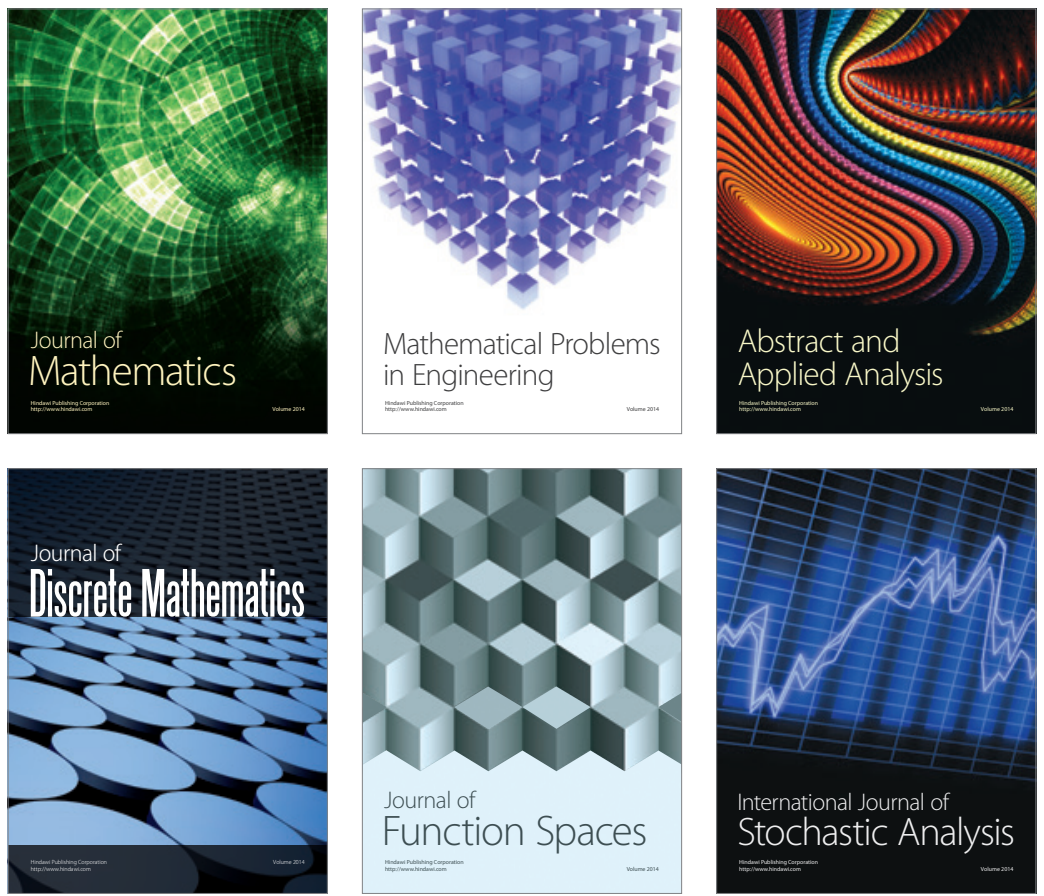

Journal of

Function Spaces

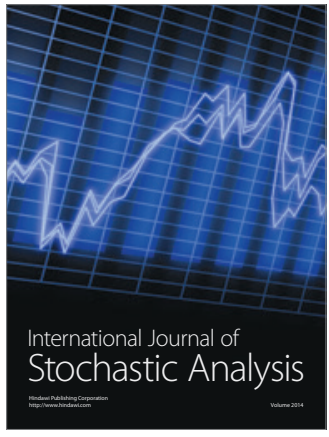

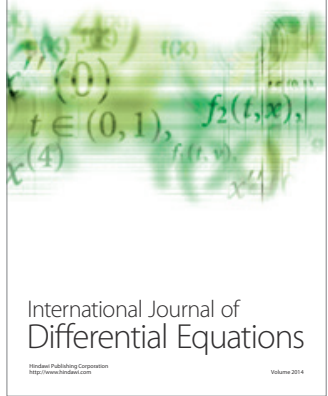
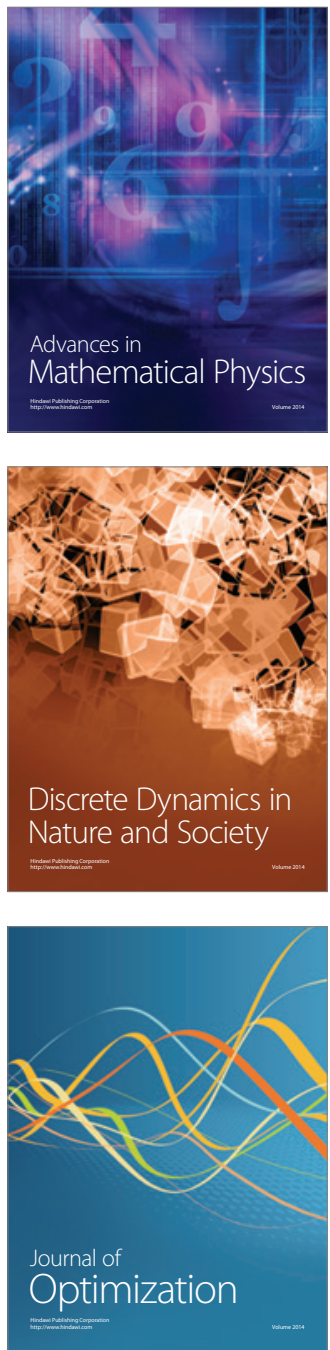